The Astrophysical Journal, 240:785-802, 1980 September 15

(C) 1980. The American Astronomical Society. All rights reserved. Printed in U.S.A.

\title{
PHOTOMETRIC STUDIES OF COMPOSITE STELLAR SYSTEMS. IV. INFRARED PHOTOMETRY OF GLOBULAR CLUSTERS IN M31 AND A COMPARISON WITH EARLY-TYPE GALAXIES
}

\author{
JAY A. FrogeL ${ }^{1}$ \\ Cerro Tololo Inter-American Observatory ${ }^{2}$ \\ S. E. Persson \\ Mount Wilson and Las Campanas Observatories, Carnegie Institution of Washington \\ AND \\ Judith G. COHEN \\ Palomar Observatory, California Institute of Technology \\ Received 1980 January 25; accepted 1980 March 19
}

\begin{abstract}
The results of an infrared photometric investigation of 40 globular clusters in and around M31 are presented. The $(V-K)_{0}$ colors of the $\mathrm{M} 31$ globulars are tightly correlated with other broadband colors and with reddening-free metallicity parameters derived from optical spectrophotometry by Searle. Over a range of $\sim 1.2 \mathrm{mag}$ in $(V-K)_{0}$, the scatter is consistent with observational error. Thus the $0.3-2.2 \mu \mathrm{m}$ energy distributions are uniquely predicted by the metallicity and vice versa. A comparison of the $(V-K)_{0}$ colors with those of galactic globulars allows an independent derivation of the metallicities of individual M31 globulars.

The broad-band infrared data are compared with predictions from integrated light models based on the Ciardullo and Demarque isochrones. The agreement is quite good for models with an initial mass function of slope $\lesssim$ the Salpeter value independent of metallicity, thus ruling out the possibility that a late-type dwarf component is making a significant contribution to the infrared light. $\mathrm{CO}$ and $\mathrm{H}_{2} \mathrm{O}$ indices measured for eight and seven of the clusters, respectively, give the same result.

Early-type galaxies are seen to have much redder broad-band colors and stronger $\mathrm{CO}$ and $\mathrm{H}_{2} \mathrm{O}$ indices than the most metal-rich M31 or galactic globular observed. Compared to the reddest globular clusters, at a given $(U-V)_{0}$ early-type galaxies are on average 0.3 mag redder in $(V-K)_{0}$. Although the stellar synthesis models reproduce cluster broad-band colors reasonably well, they do not reproduce the $U-V / V-K$ distribution of early-type galaxies. We propose that the early-type galaxies contain a population of cool luminous stars present neither in the clusters nor in the stellar synthesis models. One candidate for this population is a giant branch of stars considerably more metal rich than the Sun. More interesting is the possibility that there is a contribution to the integrated infrared light from asymptotic giant-branch stars above the first red giant tip. Such stars could be of intermediate age.

The luminosity functions for the M31 and the galactic globulars are examined with the aid of models to investigate the possibility that metal-enhanced star formation or variations in the initial mass function can be detected in integrated light.

Two appendices present new infrared data for a faint $\mathrm{dE}$ galaxy in the Virgo cluster, and a recalibration of the integrated light models presented by Aaronson et al.

Subject headings: clusters: globular - galaxies: individual -- galaxies: photometry galaxies: stellar content - infrared: sources
\end{abstract}

\section{INTRODUCTION}

Globular clusters have the potential to help us understand the stellar population and dynamical and

\footnotetext{
${ }^{1}$ Guest Investigator at the Hale Observatories, 1976-1978, and at Kitt Peak National Observatory, which is supported by the National Science Foundation under contract AST 78-27879.

${ }^{2}$ Cerro Tololo Inter-American Observatory is supported by the National Science Foundation under contract AST 78-27879.
}

chemical evolution of galaxies. Since single globular clusters are coeval and chemically homogeneous, they provide an attractive base on which to test synthesis models for the composite light of more complex systems such as elliptical galaxies which contain billions of stars and undoubtedly a wide range in metal abundance. Their usefulness in tracing the metalenrichment sequence and early history of our Galaxy has recently been exploited by Zinn (1980), Searle and 
Zinn (1978), Cowley, Hartwick, and Sargent (1978), and Canterna and Schommer (1978).

In this paper we present and discuss infrared data for a sample of globular clusters around the nearby spiral M31. The efficacy of such data for investigating the stellar content of globular clusters and elliptical galaxies has previously been demonstrated (Frogel et al. 1978; Aaronson, Frogel, and Persson 1978, hereafter Papers I and II; Frogel, Persson, and Cohen 1978; Aaronson et al. 1978, hereafter ACMM; Cohen, Frogel, and Persson 1978, hereafter CFP; Strom et al. 1976). The basic observed parameters-broad-band JHK colors and $\mathrm{CO}$ and $\mathrm{H}_{2} \mathrm{O}$ absorption indices - are sensitive to bolometric luminosity and the location in effective temperature of the composite giant branch which is related to the mean metal abundance. They can set limits on the relative numbers of giants and dwarfs and on allowable giant-branch luminosity functions.

The M31 cluster data, corrected for reddening according to the precepts of Searle (1980), are used to investigate the intrinsic infrared properties of the M31 cluster system and are compared with similar data for early-type galaxies. We first show that the broad-band $V-K$ colors of the M31 clusters are well correlated with Searle's metallicity parameters, and then use ACMM's observations of galactic globulars to determine the relative metallicities of the M31 clusters. This provides a calibration of Searle's reddening-free parameters in terms of $[\mathrm{Fe} / \mathrm{H}]$.

Next, we compare the infrared properties of the clusters with those of early-type galaxies. Faber (1973, 1977) has argued that elliptical galaxies form a "smooth, natural extension of the globular cluster sequence in all respects" for any combination of color or line strength. She, Strom et al. (1976), and ACMM have attempted to use the dependence of various photometric indices on metallicity determined from galactic globulars to infer the metallicity of $\mathrm{E}$ and $\mathrm{S} 0$ galaxies. Infrared colors and indices offer a new way to compare these systems, as the mechanisms which control the changes from cluster to cluster and from galaxy to galaxy produce different effects in the infrared than at visible wavelengths. The basic question we seek to answer is whether we can construct galaxies of composite metal abundance from the light of individual globular clusters each with a unique metal abundance. If not, can we characterize what is present in the galaxies but not in the globulars as a function of wavelength?

Finally, the M31 cluster data are used, with the aid of the population models, to look at the initial mass function (IMF) of the clusters. Freeman (1977) has reviewed some problems in the determination of the IMF for stellar population models of galaxies and has stressed the usefulness of globulars in this context since one can determine the IMF for a number of them via their color magnitude diagrams and star counts. We also briefly discuss the luminosity functions of the integrated light of the Milky Way and M31 globular clusters.
Some of the results of this work have already appeared in a preliminary form (Frogel, Persson, and Cohen 1978).

\section{THE DATA}

\section{a) Observations}

All of the data presented in this paper were obtained on the $5 \mathrm{~m}$ telescope during the fall observing seasons of 1976, 1977, and 1978. The filters, detector system, and observing techniques were essentially the same as those described in Papers I and II and in CFP. Many of the observations from all of these programs were in fact obtained on the same nights.

Initially we attempted to secure broad-band $J H K$ data for all clusters in M31 which were classified as globular by van den Bergh (1969), with $V<16.5$, and whose locations did not seem to present crowding problems on the basis of inspection of a large-scale photograph of M31. All clusters were easily visible in the photometer eyepiece; visual centering was checked by centering the $1.65 \mu \mathrm{m}$ signal as well. The "sky" points were located symmetrically north and south of each cluster at a typical distance of $20^{\prime \prime}$. Since we were chopping and beam switching, any uniform background from the M31 disk was automatically removed from the observations, while any gradient in the background greater than $5 \%$ between star and sky beams would have been apparent in the raw data. Five of the brightest, and presumably most extended, clusters were observed with both a $15^{\prime \prime}$ and a $10^{\prime \prime}$ diameter aperture. The magnitudes through the smaller aperture were only $\sim 0.04$ fainter in the mean than those measured with the larger aperture. Since this is no larger than a typical photometric error, no corrections have been applied to the $K$ magnitudes for purposes of comparing them with the $V$ magnitudes which have been obtained with aperture sizes between $12^{\prime \prime}$ and 20" (e.g., van den Bergh 1969).

Columns (1) and (2) of Table 1 give the cluster identification numbers from Vetesnik (1962) and Sargent et al. (1977), respectively. Column (6) indicates the aperture size through which the $K$ magnitude (column [7]) was measured. Broad-band infrared colors are given in columns (8) and (9). We give only the reddening-corrected (see below) values for the $\mathrm{H}_{2} \mathrm{O}$ and $\mathrm{CO}$ indices.

Whenever possible, photoelectric $U B V$ values were obtained from the compilation of van den Bergh (1969). Otherwise, we obtained our own $U B V$ data with the KPNO $1.3 \mathrm{~m}$ telescope and the Mark I photometer. Standard pulse-counting techniques were used and generally four different sky positions were measured for each object. In the absence of any photoelectric photometry, photographic $U B V$ values from the compilation of Harris (1974) were adopted as noted in Table 1. 


\section{b) The Reddening Values}

Previous studies of the M31 globular clusters have assumed either a uniform reddening for the clusters (e.g., van den Bergh 1969) or a mean unreddened color for all the clusters (e.g., Harris 1974). By combining our infrared data with optical photometry and several reddening-free metallicity indices, Searle (1980) has been able to determine reddening values for each cluster individually. His derived values for $E(V-K)$ are given in column (10) of Table 1 . We will not repeat here a description of the technique he used, but merely note two points. Those clusters in Table 1 with $E(V-K)=0.28$ (corresponding to $E[B-V]=0.10)^{3}$ are not reddened by material internal to $\mathrm{M} 31$, but only by foreground material in our own Galaxy. Second, owing to lack of significant evidence to the contrary, a normal galactic reddening law was assumed (van de Hulst's curve No. 15, Johnson 1966a). The reddening and extinction-corrected magnitudes, colors, and narrow-band indices are given in columns (11)-(17) of Table 1. Finally, we tabulate two of Searle's reddeningfree metallicity parameters in columns (18) and (19). These parameters will be discussed fully by Searle (1980).

\section{A COMPARISON OF THE M31 AND GALACTIC GLOBULAR CLUSTERS}

Our objective in this section is to derive a metallicity calibration for the M31 clusters on the basis of the new infrared data. Reasons for expecting the integrated $\mathrm{CO}$ indices and $(V-K)$ colors of globular clusters to be functions of metallicity have been reviewed by Frogel, Persson, and Cohen (1978) and ACMM, and in Paper I. Since we will derive this calibration from a colormetallicity relationship for galactic globulars, it is necessary to establish that the M31 and galactic globulars have similar energy distributions and narrow-band indices, and also that these energy distributions are tightly correlated with a reliable metallicity parameter for integrated light.

\section{a) Colors of M31 and Galactic Globular Clusters}

Figures $1 a, 2 a, 3$, and 4 present the basic color-color and color-index correlations for the M31 and galactic globulars. The galactic globular data were obtained by ACMM, who have kindly given us access to their data. For the galactic globulars we have recalculated reddening-corrected values for the infrared colors and indices from the $E(B-V)$ values of Zinn (1980) and Harris (1976). Figure $1 a$ shows a tight correlation of $(V-K)_{0}$ with $(U-V)_{0}$ over a range $>1$ mag in each color; the dispersion in the relationship is consistent with that expected from observational error. The two cluster systems overlap completely in this plot, any

\footnotetext{
${ }^{3}$ This value is a mean of those determined by van den Bergh (1969) of $E(B-V)=0.09 \pm 0.02$ and by McClure and Racine (1969) of $E(B-V)=0.11 \pm 0.02$.
}

weighting of the distributions being due to selection effects or to the difficulty of observing metal-rich clusters in the plane of our Galaxy. Figure $2 a,(J-K)_{0}$ versus $(U-V)_{0}$, also shows a correlation that allows little or no scatter exceeding observational error. The broad-band energy distributions of the M31 and galactic globular clusters are thus indistinguishable, and both distributions change smoothly along the color sequence. A cluster that is "blue" over some wavelength interval is "blue" everywhere.

Figure 3 shows that the $\mathrm{CO}$ indices of the two cluster systems overlap and that there is an obvious correlation between broad-band color and $\mathrm{CO}$ strength. The $\mathrm{H}_{2} \mathrm{O}$ data plotted in Figure 4 are not as clear-cut, but there is a suggestion of a correlation between $\mathrm{H}_{2} \mathrm{O}$ and $\mathrm{CO}$.

In Figure 5 we plot the energy distributions of the M31 globulars, represented here by the $(V-K)_{0}$ colors, against Searle's (1980) $Q_{K}$ parameter. This parameter measures, in a reddening-free manner, the depression of the flux at $\lambda 3880$ below an extrapolated continuum and thus includes the Calcium $\mathrm{H}$ and $\mathrm{K}$ lines. (The other reddening-free parameter, $q$, is similar to $Q_{K}$ except that it measures the $\lambda 3880$ band depression with respect to a locally determined continuum and is independent of the reddening law.) The $Q_{K}$ parameter is basically the same quantity, to within a well-defined transformation, as Zinn's (1980) parameter $Q_{39}$ which was demonstrated by Zinn to be very tightly correlated with $[\mathrm{Fe} / \mathrm{H}]$ for galactic globular clusters. Figure 5 shows that $(V-K)_{0}$ is very tightly correlated with $Q_{K} \cdot{ }^{4}$ Thus we have the result that the energy distributions of the M31 globular clusters are correlated one-to-one with heavy metal abundance. This correlation surely arises from the shift redward of the giant branch with increasing metal abundance. What is remarkable is that the observed ccrrelation allows little or no cosmic scatter which might arise, for example, from variations in horizontal-branch morphology, stochastic effects, or variations in the relative numbers of late-type dwarfs which can strongly affect $(V-K)_{0}$. We shall return to these points later.

Finally, we consider van den Bergh's (1969) linestrength parameter $L$ which is the one metallicity parameter presently available for both the M31 and galactic globular systems. Figure 6 displays this parameter as a function of $(V-K)_{0}$ for the two cluster systems. Although there are few metal-rich galactic globulars having infrared photometry, we see that the two cluster systems follow the same relationship between $L$ and $(V-K)_{0}$. This does not imply, of course, that there are no differences in the distribution over metallicity between the M31 globulars and the galactic ones (van den Bergh 1967, 1969; Hanes 1977; Searle 1978)

\footnotetext{
${ }^{4}$ For the 10 clusters of Table 1 with $E(V-K)>0.28$, the reddening values were derived from the relationship between $(V$ $-K)$ and $q$ for clusters with no reddening due to dust internal to M31 (cf. Searle 1980). Removal of these 10 clusters does not significantly alter the tightness of the relation in Figure 5.
} 


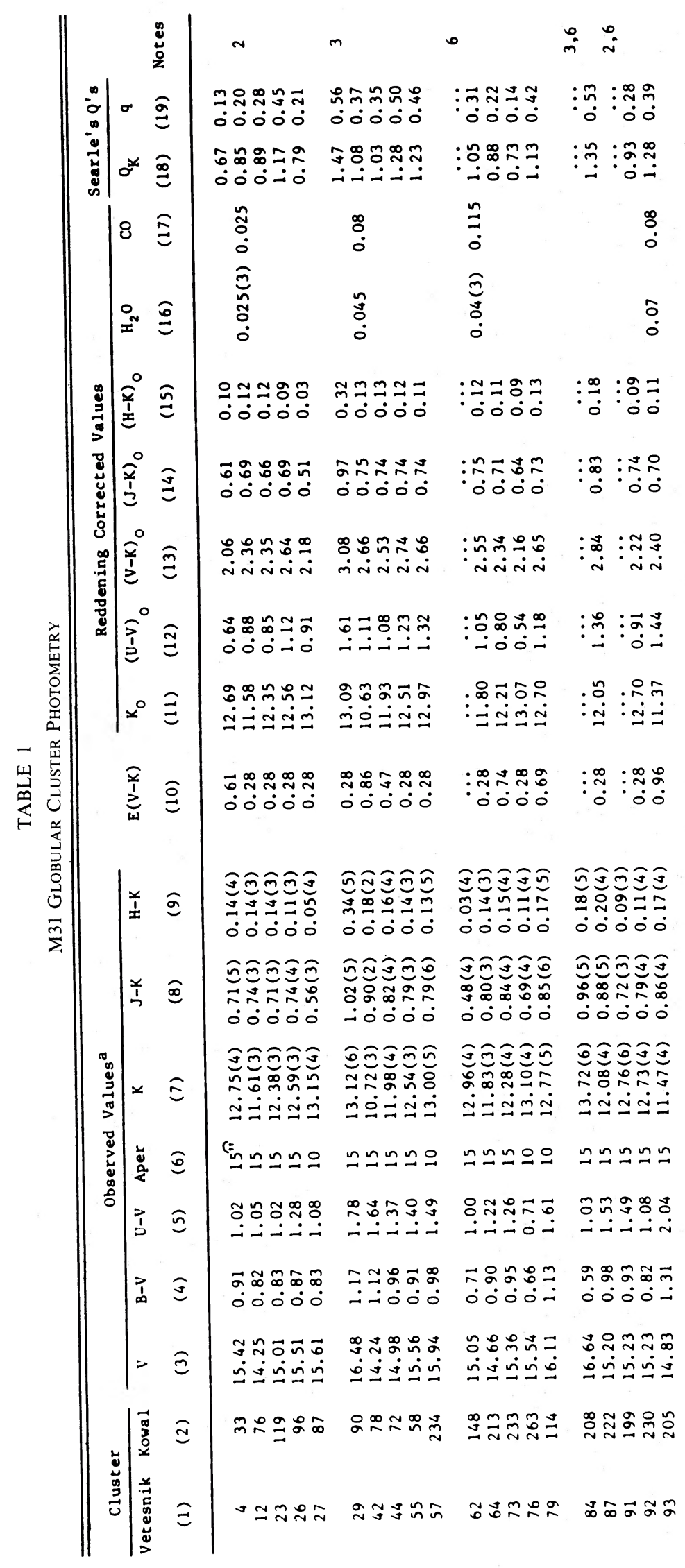

788

(C) American Astronomical Society - Provided by the NASA Astrophysics Data System 


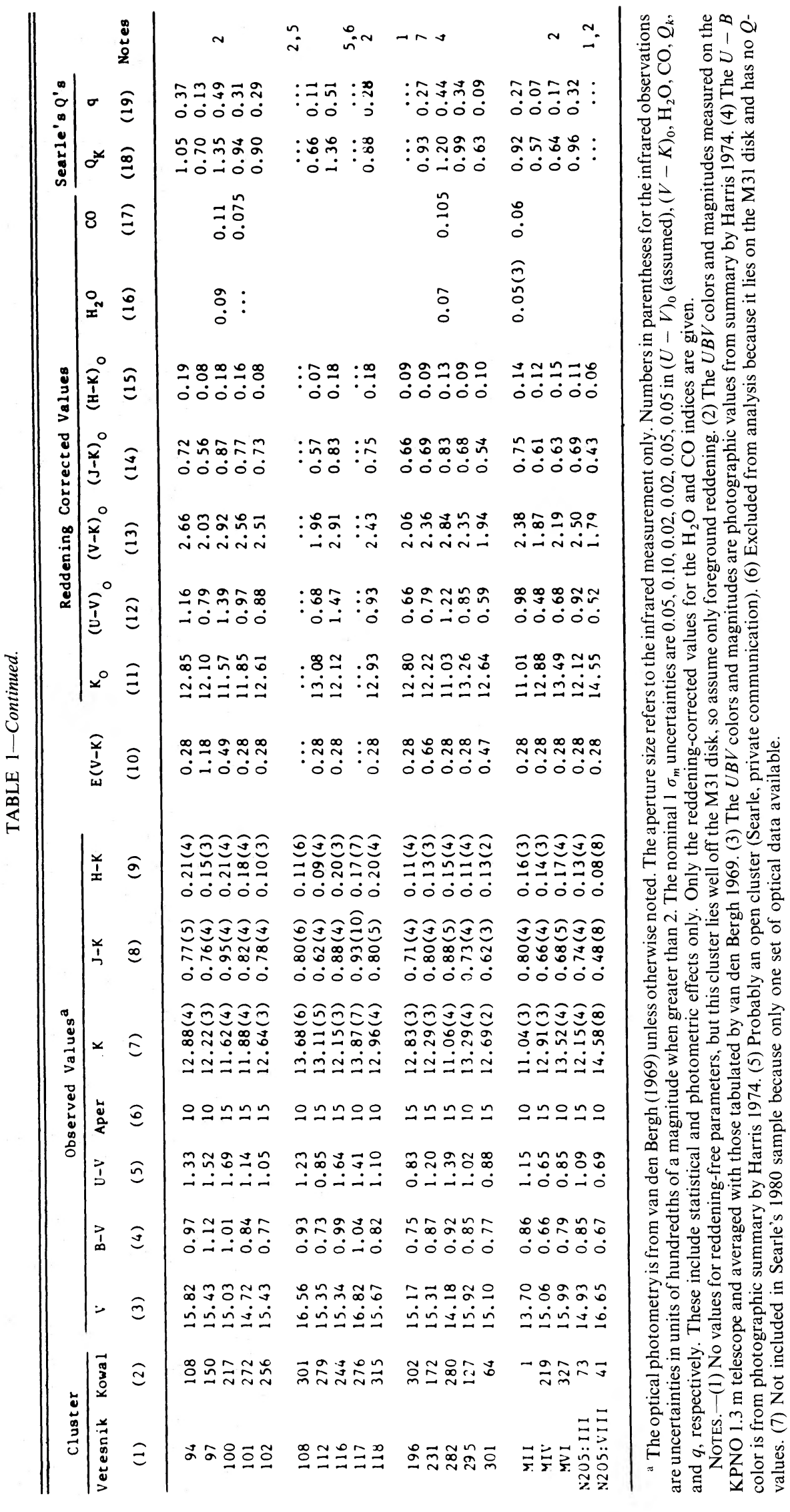




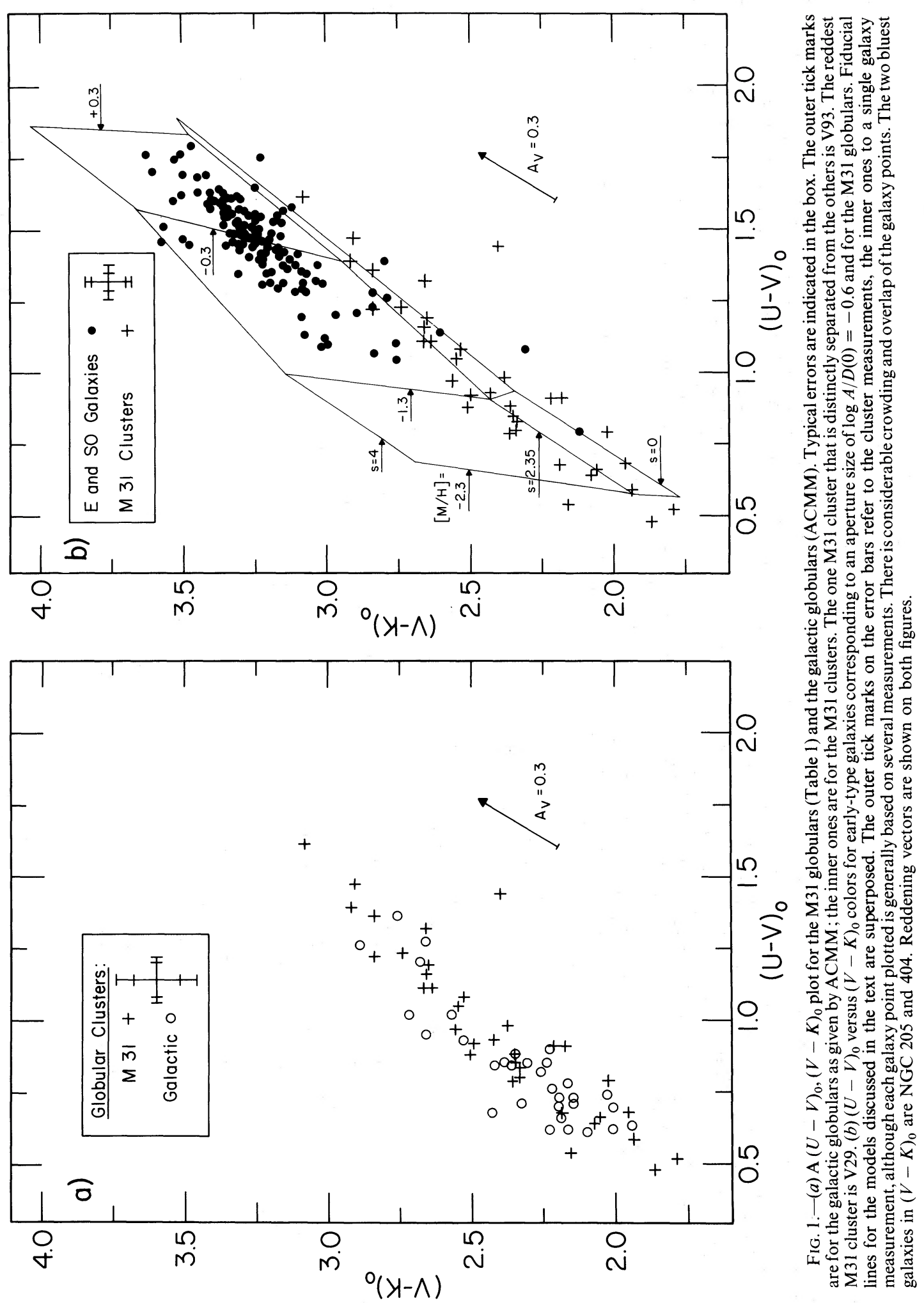




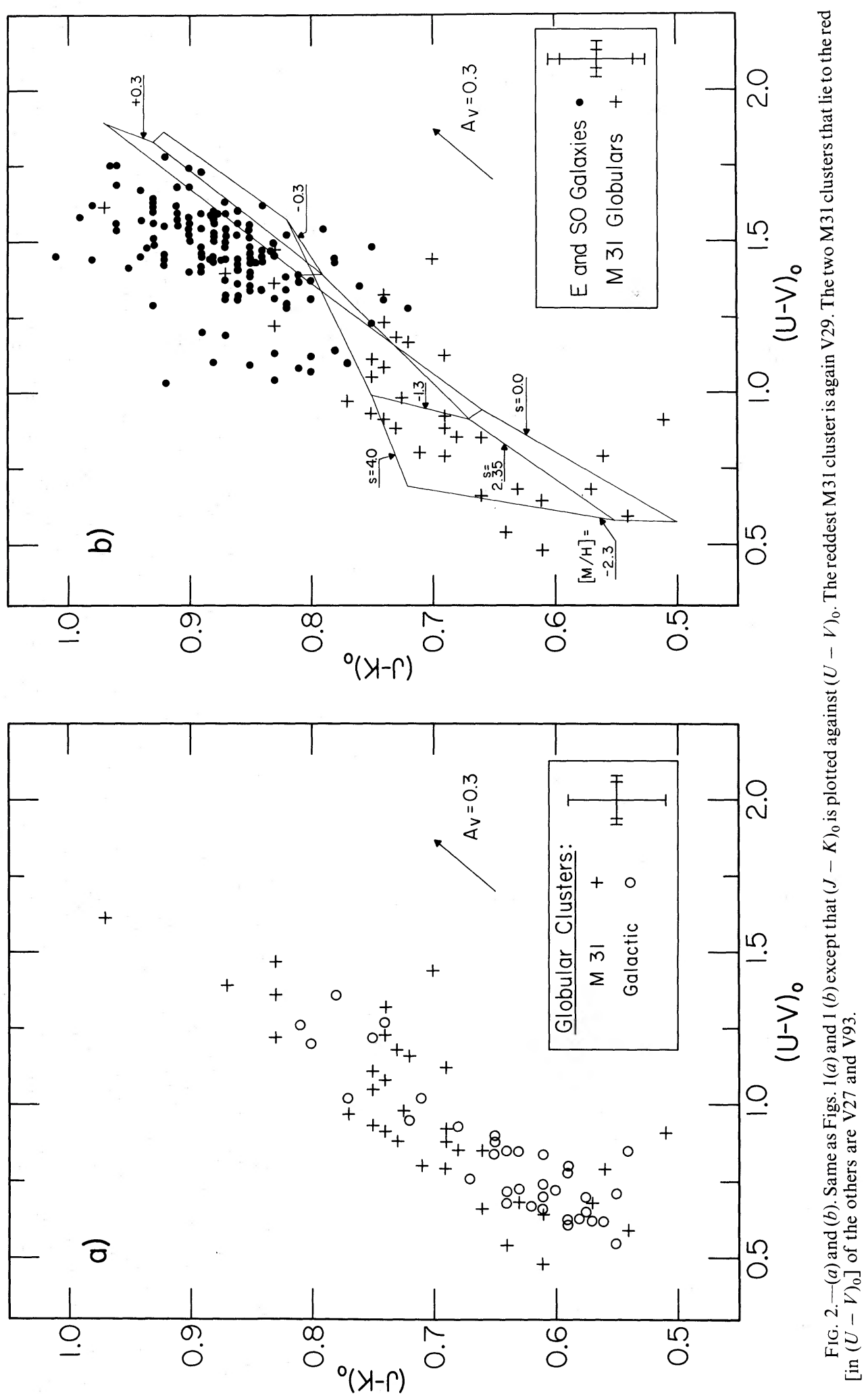




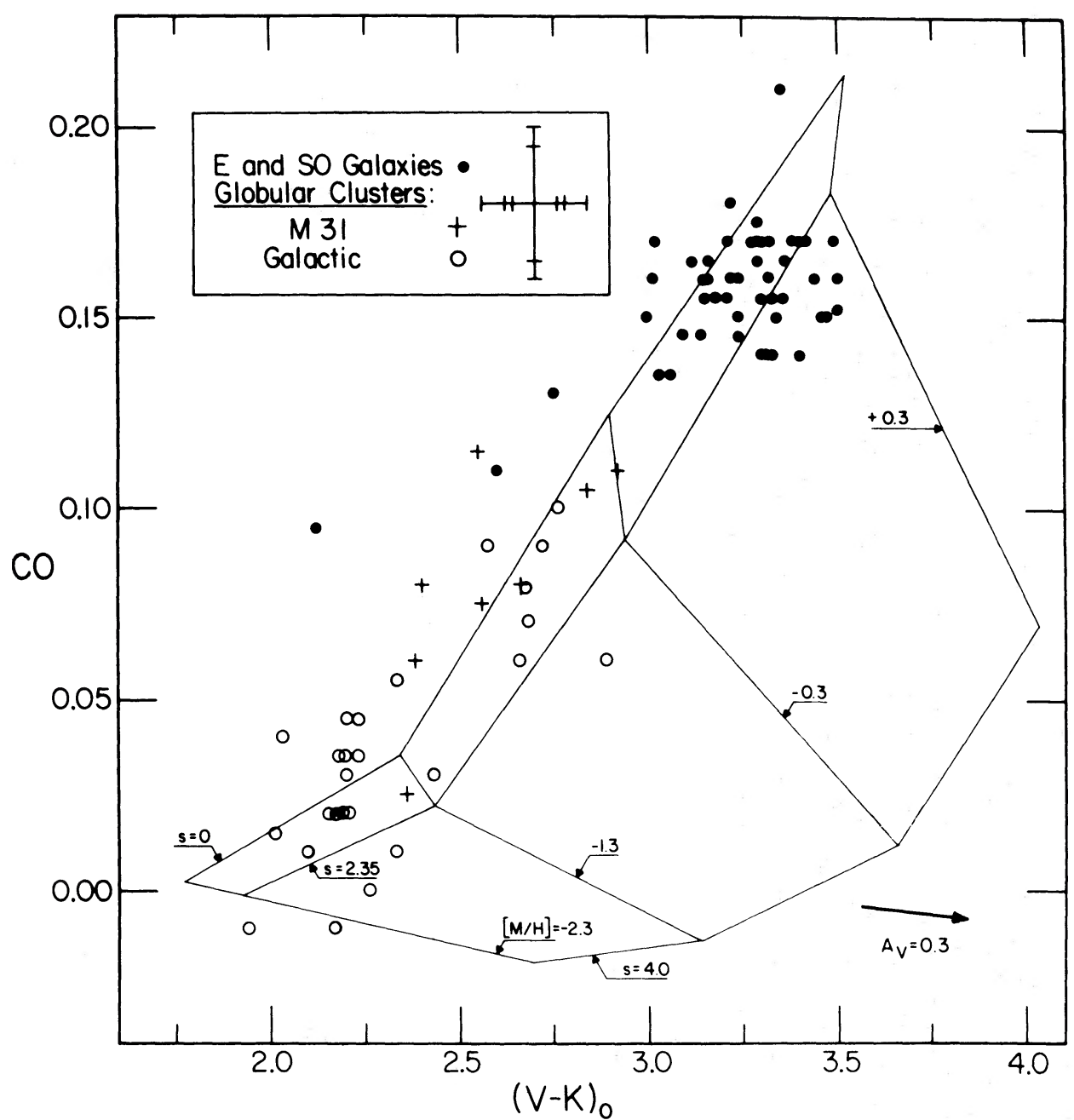

FIG. 3.-A CO, $(V-K)$ plot for M31 and galactic globulars and for early-type galaxies from Paper I, Persson et al. (1980b), and Appendix A The outermost tick marks on the error bars are typical for the galactic globulars (ACMM) and, in CO, for the M31 clusters. The middle set of tick marks in $(V-K)_{0}$ are also for the M31 clusters. The innermost tick marks are for the galaxies where CO values are in most cases an average over several different aperture sizes. The $(V-K)_{0}$ values for the galaxies are those for an aperture corresponding to $\log A / D(0)=-0.6$. The three bluest galaxies in order of decreasing $(V-K)_{0}$ are M100 chart $1, \mathrm{NGC} \mathrm{404,} \mathrm{and} \mathrm{NGC} \mathrm{205.} \mathrm{The} \mathrm{point} \mathrm{at} \mathrm{CO}=0.21$ is a single measurement of NGC 6702 .

We can now proceed with some degree of confidence to transfer a metallicity-color calibration for galactic globulars to the M31 globulars.

\section{b) The Metallicity Calibration}

To place the M31 clusters on a metallicity sequence, we plot in Figure 7 the $(V-K)_{0}$ and $\mathrm{CO}$ data for galactic globulars against the $[\mathrm{Fe} / \mathrm{H}]$ values derived from the work of Zinn (1980). Formal least-squares solutions for the dependences of $(V-K)_{0}$ and $\mathrm{CO}$ on $[\mathrm{Fe} / \mathrm{H}]$ were found by averaging the two solutions obtained by taking first the metallicity and then the observed color as the independent variable. We find

$$
\begin{aligned}
(V-K)_{0} & =2.96+0.43[\mathrm{Fe} / \mathrm{H}]_{\text {Zinn }}, \\
\mathrm{CO} & =0.110+0.048[\mathrm{Fe} / \mathrm{H}]_{\text {Zinn }},
\end{aligned}
$$

with $1 \sigma$ residual in $[\mathrm{Fe} / \mathrm{H}]$ of 0.28 and 0.38 , re- spectively. The dashed lines in Figure 7 are these $1 \sigma$ values at a given observed value of $(V-K)_{0}$ or $\mathrm{CO}$. Although based on a sample size more than twice as large as the ACMM calibrators and somewhat different input parameters, equations (1) and (2) are in essential agreement with the ACMM results. Both sets of results are heavily weighted toward clusters with metal deficiencies compared to the Sun of a factor of 20 or more.

An important point is that the $1 \sigma$ residuals in the values of $[\mathrm{Fe} / \mathrm{H}]$ are comparable to the uncertainties expected from the observational uncertainties alone $\left(0.10-0.15 \mathrm{mag}\right.$ in $[V-K]_{0}$ and $0.02 \mathrm{mag}$ in $\left.\mathrm{CO}\right)$. These uncertainties are particularly large in the case of galactic globulars because of mismatch between the optical and infrared aperture sizes, centering, background contamination, and some uncertain reddenings. The size of the residuals implies that to the 


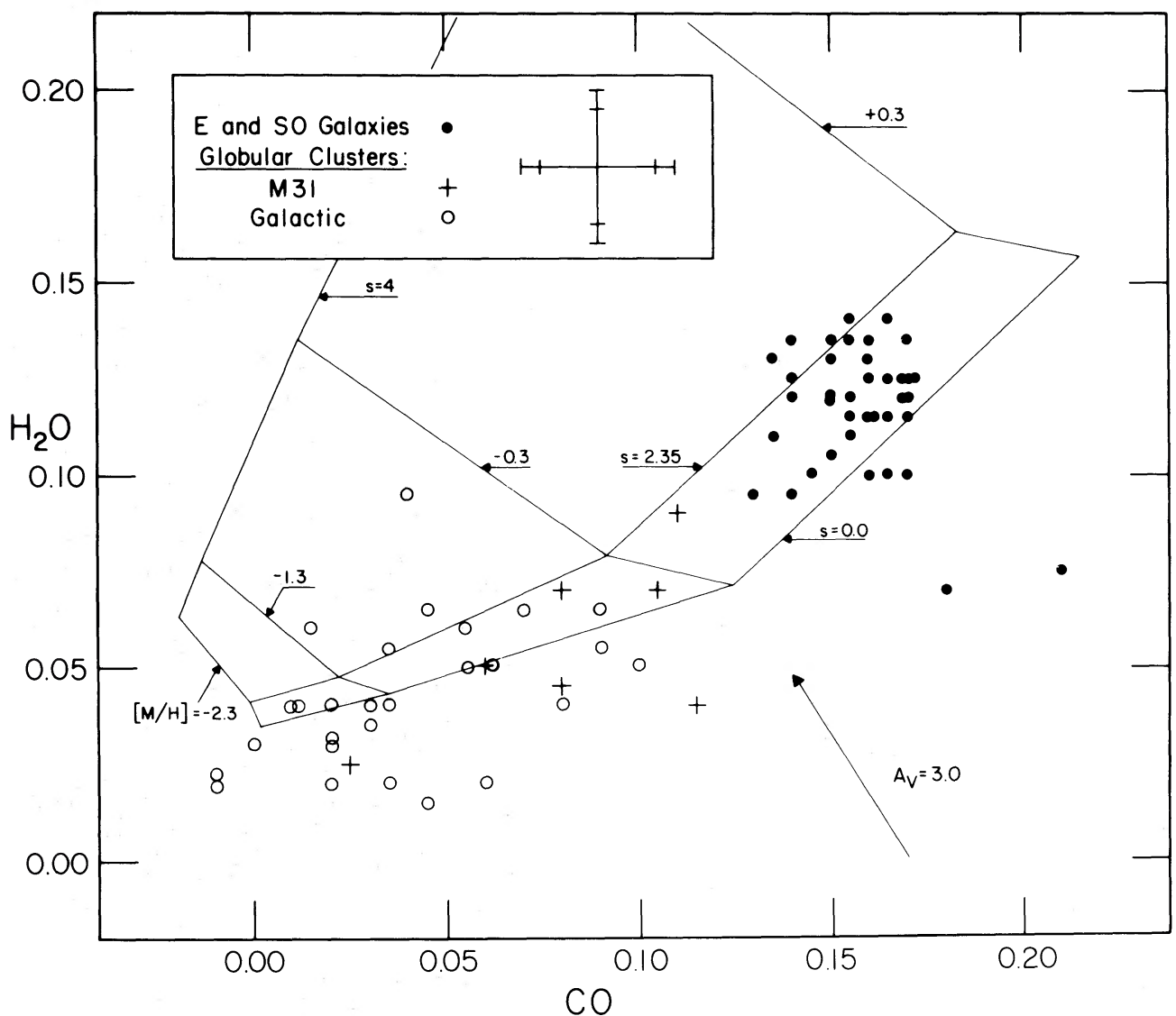

Fig.-Similar to Fig. 3 except with $\mathrm{H}_{2} \mathrm{O}$ against $\mathrm{CO}$. The description of the tick marks on the $\mathrm{CO}$ error bar of Fig. 3 is appropriate to both error bars here. The two galaxy points with the lowest $\mathrm{CO}$ values are single measurements of NGC 4283 and NGC 6702 . M100 CH 1 is the galaxy with the weakest $\mathrm{CO}$ of the main group of galaxies. Note the difference in scale of the reddening vector compared with the previous figures.

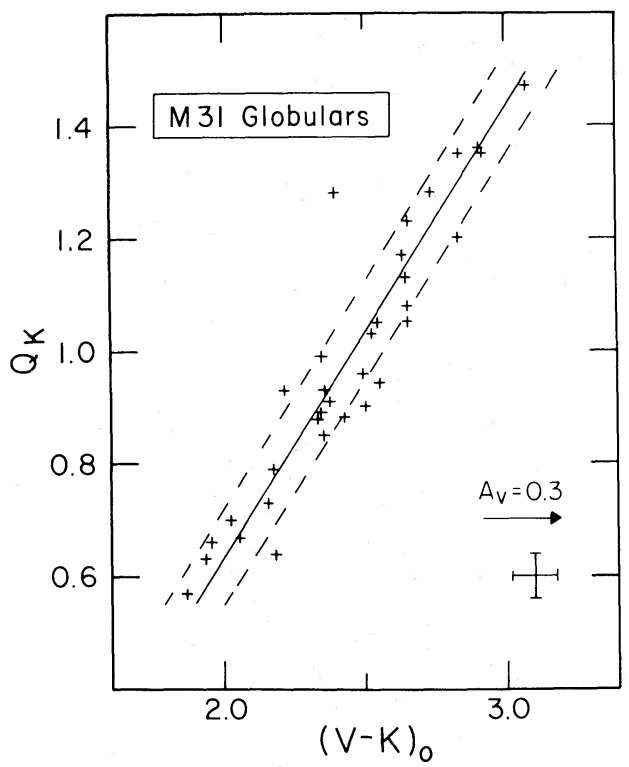

FIG. 5.-Searle's (1980) reddening-free metallicity parameter plotted against $(V-K)_{0}$ for the M31 clusters. The best-fit leastsquares lines and the $\pm 1 \sigma$ residuals are also indicated as discussed in the text. A typical error bar and a reddening vector are in the lower right. The one cluster separated from the rest is again V93.

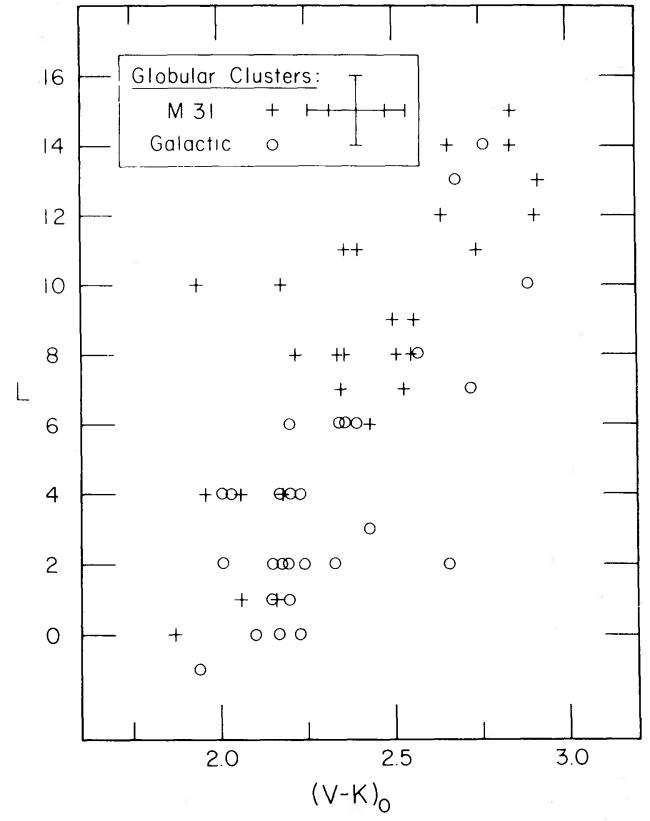

FIG. 6.-Van den Bergh's (1969) $L$ indices plotted against $(V-K)_{0}$ colors for M31 and galactic globulars. The inner tick marks on the $V(V-K)_{0}$ error bar refer to the M31 clusters. The error in the $L$ indices has been arbitrarily taken as \pm 1 . 


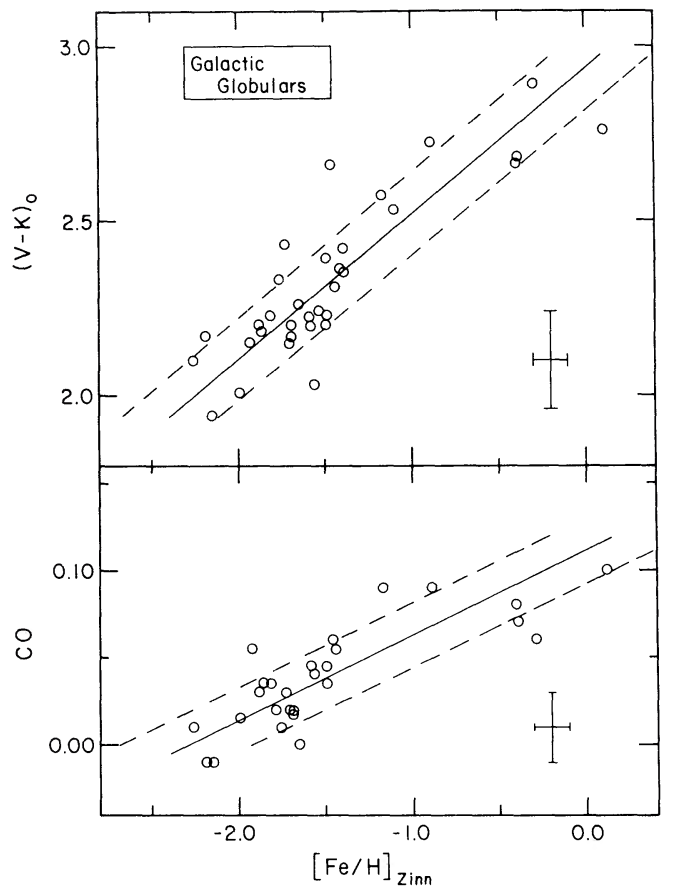

FiG. 7. - The relationship between $(V-K)_{0}, \mathrm{CO}$, and $[\mathrm{Fe} / \mathrm{H}]$ for galactic globular clusters. The $(V-K)_{0}$ and $\mathrm{CO}$ values are adapted from ACMM; the $[\mathrm{Fe} / \mathrm{H}]$ values are from Zinn (1980). Best-fit least-squares solutions and the $\pm 1 \sigma$ residuals discussed in the text are also shown.

accuracy of ACMM's measurements and Zinn's metallicity calibration there is little or no cosmic scatter in the dependence of $(V-K)_{0}$ or the CO index on metallicity for galactic globulars.

From the data in Figure 5, the formal least-squares solution for $Q_{K}$ as a function of $(V-K)_{0}$ for the M31 sample is obtained by averaging the solutions derived by taking the two quantities in turn as the independent variable:

$$
Q_{K}=-0.97+0.80(V-K)_{0} .
$$

The $1 \sigma$ residual in $(V-K)_{0}$ is \pm 0.11 , again comparable to that expected from the measuring errors alone. From equations (1) and (3) we obtain

$$
Q_{K}=1.40+0.34[\mathrm{Fe} / \mathrm{H}]_{\text {Zinn }} \text {. }
$$

Equation (4) will yield metallicity values for the 200 M31 clusters for which Searle (in preparation) has $Q_{K}$ values. The $1 \sigma$ uncertainty in a value of $[\mathrm{Fe} / \mathrm{H}]$ determined from $Q_{K}$ will be $\pm 0.36{ }^{5}$

Searle (1980) notes that from $U$ to $K$ "the spectral flux distributions of the integrated light of the M31 clusters form a one-parameter family." The above results imply that the one parameter needed to rank the

\footnotetext{
${ }^{5}$ Equation (4) implicitly assumes that the M31 and galactic globular cluster systems have the same age and stellar lumnosity function. An age difference of $5 \times 10^{9}$ years, however, would cause a difference of only $0.1 \mathrm{mag}$ in $(V-K)_{0}$ between two clusters with the same metallicity and luminosity function, according to the calculations of Struck-Marcell and Tinsley (1978; but see $\S$ V below)
}

spectral flux distributions from $U$ to $K$ of the integrated light of the M31 clusters is heavy metal abundance.

Recently, Cohen (1979) and Pilachowski, Canterna, and Wallerstein (1980) have given evidence that the "metal rich" globular clusters M71 and 47 Tucanae are actually moderately metal poor. The change implied by their detailed abundance analysis takes these clusters from -0.4 to $\sim-1.1$ in $[\mathrm{Fe} / \mathrm{H}]$. The effect of such a change brings about a drastic alteration in Zinn's (1980) metal-abundance scale, and it appears quite possible that his correlation of $Q_{39}$ with $[\mathrm{Fe} / \mathrm{H}]$ will become nonlinear near $[\mathrm{Fe} / \mathrm{H}]_{\text {Zinn }}=-1$. The relative ranking of the clusters is unaffected, of course. Rather than propose a new scale here we merely caution that equations (1), (2), and (4) apply only to Zinn's present scale and will probably change when fundamental metal abundances are available for an extended sample of stars and clusters.

The range in metal abundance that we derive for the M31 globulars is in qualitative agreement with the results of van den Bergh (1969) and Spinrad and Schweizer (1972), but we do not agree with Spinrad and Schweizer that this is a wider range than is found for galactic globulars even though we and they have observed the same metal-rich M31 clusters (see also Harris and Canterna 1977). Comparing the upper bound of the values of $(V-K)_{0}$ for the two samples, we see that when allowance is made for selection effects (most of the metal-rich galactic-center clusters were not observed by ACMM), plus uncertainties in the metallicity determinations, there is probably no significant difference in the upper bound to the metallicity distribution for the two systems of globulars. In particular, we note that clusters 87, 282, and MII, which Spinrad and Schweizer claimed had no galactic counterpart, have energy distributions similar to the reddest galactic globulars for which data were available from ACMM. Cluster 29, in fact, is the M31 cluster with the reddest colors and largest $Q_{K}$ value. It remains to be seen if some of the M31 clusters not included in the present program (nor in those of van den Bergh 1969 and Spinrad and Schweizer 1972) do in fact extend this upper bound.

In Table 2 we tabulate the metallicities determined from equations (1) and (2) for those M31 clusters having both $\mathrm{CO}$ and $(V-K)_{0}$ data or having a previous metallicity determination by Harris and Canterna (1977). The dispersion in the differences between the $\mathrm{CO}$ and $(V-K)_{0}$ values is \pm 0.44 , consistent with what would be expected from the values of the two residuals $( \pm 0.47)$. The differences between our metallicity values and those of Harris and Canterna are large but consistent with similar differences found by Searle and Zinn (1978) and Zinn (1980) for galactic globulars observed on the $\mathrm{CMT}_{1} \mathrm{~T}_{2}$ system and other photometric systems.

\section{c) Stochastic Effects}

The scatter about the mean lines in Figures 1 and 5 is consistent with that expected from observational un- 
TABLE 2

The Metallicities of Selected M31 Globulars

\begin{tabular}{|c|c|c|c|c|}
\hline \multirow{2}{*}{$\begin{array}{c}\text { CluUSTER } \\
\text { VETESNIK } \\
\text { NuMBER }\end{array}$} & \multicolumn{3}{|c|}{$[\mathrm{Fe} / \mathrm{H}]$ FROM INFRARED } & \multirow[b]{2}{*}[\mathrm{Fe}/\mathrm{H}]{$_{\mathrm{HC}}{ }^{b}$} \\
\hline & $(V-K)_{0}$ & $\mathrm{CO}$ & Mean $^{a}$ & \\
\hline$\ldots \ldots$ & -1.39 & -1.77 & -1.55 & -0.9 \\
\hline 42. & -0.68 & -0.63 & -0.66 & -0.6 \\
\hline $44 \ldots$ & -0.98 & & -0.98 & -0.4 \\
\hline 64. & -0.94 & 0.10 & -0.50 & $\ldots$ \\
\hline $93 \ldots$ & -1.29 & -0.63 & -1.01 & \\
\hline $100 \ldots \ldots \ldots$ & -0.06 & -0.11 & -0.08 & \\
\hline $101 \ldots \ldots \ldots$ & -0.91 & -0.73 & -0.83 & -0.8 \\
\hline $282 \ldots \ldots \ldots \ldots$ & -0.25 & -0.11 & -0.19 & 0.0 \\
\hline M II $\ldots \ldots \ldots$ & -1.34 & -1.05 & -1.22 & -0.3 \\
\hline
\end{tabular}

${ }^{a}$ The mean value is determined by weighting the $\mathrm{CO}$ and $(V$ $-K)_{0}$ determinations with the inverse of the respective residuals.

${ }^{b}$ These are the values from Harris and Canterna 1977.

certainties alone. ACMM calculated, from Monte Carlo simulations, that at a $M_{K_{0}}$ of -10.3 , one would expect an intrinsic dispersion of $\pm 0.10 \mathrm{mag}$ in $(V$ $-K)_{0}$ and $\pm 0.02 \mathrm{mag}$ in $(U-V)_{0}$. This comes about because the infrared energy output of the clusters is dominated by a small number of giant stars, while the ultraviolet comes from substantial numbers of turn-off stars, dwarfs, and subgiants. This dispersion is not seen in our data (Figs. 1 and 5), and we conclude that the cosmic scatter is even smaller at the faint end than would be deduced at first sight. For the more massive clusters, the stochastic effects are expected to drop as the square root of the total mass; for these clusters some cosmic scatter cannot be completely ruled out by the data. The importance of dealing with stochastic effects even in systems with as many as $10^{5}$ stars has been noted by Ciardullo and Demarque (1978).

\section{COMPARISON OF THE PHOTOMETRIC PROPERTIES OF} GLOBULAR CLUSTERS AND EARLY-TYPE GALAXIES

As old stellar systems which have evolved to approximately the same age, $10^{10}$ years, globular clusters and early-type galaxies share a basic similarity. In this section we compare the integrated infrared light of the two types of systems. Then in $\S \mathrm{V}$ stellar synthesis models are used to interpret the M31 observations and to examine color differences between the globulars and the galaxies. It is important to bear in mind that the galaxy data typically refer to measurements with an aperture corresponding to $\log A / D(0)=-0.6$ on the system of the Second Reference Catalogue of Bright Galaxies (de Vaucouleurs, de Vaucouleurs, and Corwin 1976). Thus the infrared measurements are averages over one-third of the visible light of the galaxies.

Figure $1 b$ is the $(U-V)_{0},(V-K)_{0}$ plot for the M31 globulars with data for early-type galaxies from Paper I, Persson, Frogel, and Aaronson (1979, hereafter Paper III), and Persson et al. (1980b). Previous results (e.g., Faber 1973, 1977) would lead one to believe that these two colors should both increase monotonically with metal abundance. Although the clusters overlap the blue end of the galaxy distribution, there are two differences between the clusters and galaxies to which we wish to draw attention. The first is that the colors of the galaxies extend to much redder values in $(U-V)_{0}$ and $(V-K)_{0}$ than the reddest globulars in either M31 or the Milky Way. Second, there is a distinct tendency for galaxies to be redder in $(V-K)_{0}$ than clusters with the same $(U-V)_{0}$. This latter result, when contrasted with the continuity of cluster and galaxy sequences at optical wavelengths (e.g., Faber 1973), illustrates that one can derive very different information at different wavelengths when dealing with a system which is highly composite in metal abundance.

The integrated $\mathrm{CO}$ absorption index as a function of $(V-K)_{0}$ for the M31 and galactic globular clusters (Table 1 here and ACMM) and for early-type galaxies (Paper I; Persson et al. 1980b; Appendix A) is shown in Figure 3 . There is a clear separation between galaxies and globulars in that galaxies have redder $(V-K)_{0}$ colors and stronger $\mathrm{CO}$ absorption. No combination of clusters, similar to those so far observed, can be constructed to reproduce the $\mathrm{CO}$ indices of galaxies. Furthermore, there is a separation between galaxies and clusters between $(V-K)_{0}=2.0$ and 3.0. At a given $(V-K)_{0}$ intrinsically faint galaxies have stronger $\mathrm{CO}$ absorption than globular clusters. The galaxy data here are very sparse, but nevertheless we believe that a real difference exists. The $\mathrm{H}_{2} \mathrm{O}$ data for the globulars and galaxies in Figure 4 (Paper II; Persson et al. (1980b), Appendix A) serve to reinforce these results. The mean $\mathrm{H}_{2} \mathrm{O}$ indices of the individual galaxies are, almost without exception, stronger than those of the globular clusters.

Finally, we consider the comparison of gaiaxies and globulars in the $(J-K)_{0},(U-V)_{0}$ plane (Fig. $\left.2 b\right)$. This figure shows a reasonably good overlap between the galaxies and globulars in contrast to the $(U-V)_{0}$ versus $(V-K)_{0}$ plot. The reason for this is simple: $(J$ $-K)_{0}$ changes only slowly with $(U-V)_{0}$ or $(V-K)_{0}$ for $(J-K)_{0}>0.8$, both for individual stars and for the clusters. (See also description of the models in $\S \mathrm{V}$.) Figure $2 b$ thus illustrates how saturation effects can alter the appearance of plots of different monotonically increasing functions of metal abundance.

\section{A COMPARISON OF THE M31 GLOBULARS AND EARLY-TYPE GALAXIES WITH MODEL PREDICTIONS}

The stellar synthesis models used in this section and drawn on in Figures $1 b, 2 b, 3$, and 4 represent a single burst of star formation evolved through $13 \times 10^{9}$ years via the Ciardullo and Demarque (1977) isochrones with modifications and additions discussed by ACMM and recomputed as discussed in Appendix B. The new models incorporate the recent temperaturescale changes brought about by the observations and 
recalibration of Tsuji (1978) and Ridgway et al. (1980) and by the revision in the metallicity of 47 Tuc discussed earlier. We note the good agreement between the models and the observations of both the M31 and the galactic globular clusters.

\section{a) The Initial Mass Function for the M31 Globular Clusters}

As ACMM found for the galactic globulars, the new stellar-synthesis models with values of $s<2.35$ reproduce the observed $(U-V)_{0},(V-K)_{0}, \mathrm{CO}$, and $\mathrm{H}_{2} \mathrm{O}$ values quite well. The insensitivity of the models does not allow us to restrict the allowable values of $s$ any further. Since the reddest M31 clusters we observed are redder than the galactic ones observed by ACMM, the restriction on the allowable values of $s$ is extended to the most metal-rich clusters.

Mass segregation effects in globular clusters might cause present-day values of $s$ appropriate to the outer regions of the clusters to be larger (perhaps as large as $s$ $=4$ ) than those for the inner regions (Da Costa 1977; reviewed by Freeman 1977). Our 15" diameter aperture used to make the M31 measurements encloses $90 \%$ or more of the total light and mass of the M31 clusters, in contrast to the situation for the galactic cluster observations. So we can extend the conclusion of ACMM and say that there is no evidence for a substantial contribution from $M$ dwarfs to the total integrated light of any of the M31 clusters; i.e., we find it unlikely that $s$ is any greater than the Salpeter value $(s=2.35)$ for all values of $[\mathrm{Fe} / \mathrm{H}]$.

\section{b) A Comparison of the Galaxy Colors with the Models}

The deviation of the galaxy sequence from that of the globular clusters and the redness of the former is emphasized in Figure $1 b$, where it is seen that the model grid, which is in good agreement with the globulars, fails to intersect the region occupied by the galaxies in the $(U-V),(U-K)$ plane for all values of these colors. Figure $2 b$ shows the same effect, namely, that at a given $(U-V)_{0}$, the galaxies are nearly 0.1 mag redder in $(J-K)_{0}$ than the models. A further difficulty that the model grid has in accounting for the galactic light is apparent in a comparison of Figures 3 and 4 with Figure $1 b$. In Figure $1 b$ the median of the distribution of galaxy points lies close to the $[\mathrm{M} / \mathrm{H}]=$ -0.3 line. In Figures 3 and 4, however, this median corresponds to an $[\mathrm{M} / \mathrm{H}]=0.0$. In other words, the $\mathrm{CO}$ and $\mathrm{H}_{2} \mathrm{O}$ indices are stronger than what one might expect from the $(U-V)_{0}$ and $(V-K)_{0}$ colors. We thus conclude on the basis of the present data that early-type galaxies show evidence for a stellar component in addition to those present in the observed globular clusters or in the models. From the $(U-V)_{0}$ versus $(V-K)_{0}$ plot alone, one can conclude only that this component is contributing an excess to either the ultraviolet or the infrared flux. An ultraviolet component could arise, for example, from a recent burst of star formation. The superposition of such a burst on the ACMM models with strength $=0.001$ as defined by Struck-Marcell and Tinsley (1978) would have the effect of keeping $(V-K)_{0}$ essentially constant, but shifting $(U-V)_{0}$ to the blue (Larson and Tinsley 1978; Struck-Marcell and Tinsley 1978). Recall, though, that the galaxy data in Figure $1 b$ refer to onethird of the total light, so that this burst would have occurred over most of the galaxy. It could not have been concentrated in the nucleus, since with few exceptions, early-type galaxies get redder in $(U-V)_{0}$ toward the nucleus. The deviation of the galactic light from the models cannot be due to the range in metal abundance within the galaxy. It is easy to show (Persson et al. 1980b) that no reasonable combination of models with different $[\mathrm{Fe} / \mathrm{H}]$ and $s \leq 2.35$ can reproduce the galaxy colors in Figure $1 b$.

One might think that the galaxy data in Figure $1 b$ could be reproduced with models having steep initial mass functions, i.e., a large relative number of cool dwarfs. The model results plotted in Figures 3 and 4, however, show quantitatively the difficulty of accounting for the colors of galaxies with dwarf-enriched light. It is not possible to construct galaxy light from any combination of clusters plus an admixture of "dwarf" light, which has strong $\mathrm{H}_{2} \mathrm{O}$ and weak $\mathrm{CO}$ (Papers I and II) because of the strengths of the $\mathrm{CO}$ and $\mathrm{H}_{2} \mathrm{O}$ features in the galaxies. This was one of the basic conclusions of Papers I and II.

What, then, is the cause for the differences between the galaxies and the globulars in Figures $1 b, 2 b, 3$, and 4 ? We believe that all these differences arise from the presence of a population of cool, luminous stars in the galaxies that are not found in clusters and that have not been included in the present models. Carbon stars cannot contribute significantly because of the constraints implied by the combination of the $\mathrm{CO}$ and $\mathrm{H}_{2} \mathrm{O}$ indices as discussed in Paper II. Reasonable fits to the $(V-K)_{0}$ colors and $\mathrm{CO}$ and $\mathrm{H}_{2} \mathrm{O}$ indices of earlytype galaxies presented in Papers I and II were achieved by the stellar synthesis models of O'Connell (1976, hereafter OC) and Tinsley and Gunn (1976a, hereafter TG). It is worthwhile to recall how such good fits to the infrared parameters were produced by these models. In the case of TG the giant-branch luminosity function was constructed from a compilation of empirical data on old disk groups (see also Tinsley and Gunn 1976b); in the case of OC, the giant-branch luminosity function, and in particular the relative populations of the coolest stellar bins (M6 and later), were unconstrained and chosen to produce consistency with the overall energy distribution and line indices. A combination of these two approaches was taken by Tinsley (1978). Both the TG and OC luminosity functions include stars well above the first red giant tip, with $M_{\text {bol }}$ as bright as -5.5 . We wish to emphasize that such stars are not present in any significant number in galactic globular clusters, nor in our stellar synthesis models. The sample of stars in well-studied globular clusters (CFP; Frogel, Persson, and Cohen 1979; 
(Persson et al. 1980a; Frogel et al. 1980) is now sufficiently large that one can assert that stars more than $0.5 \mathrm{mag}$ above the first red-giant tip at $M_{\mathrm{bol}} \sim$ -3.6 are essentially absent in old, metal-poor stellar populations having turnoff masses $<1 M_{\odot}$. This empirical result is in qualitative agreement with the theoretical calculations of Gingold (1974).

Current estimates of mass-loss rates suggest that the upper asymptotic giant branch (AGB) stars contained in the models of TG and OC can be produced only in a population where the turnoff mass is somewhat larger than $1 M_{\odot}$, as discussed for the clusters of the Magellanic clouds by Mould and Aaronson (1979). Predictions relating to the occurrence and appearance of such stars have been made by Rose and Smith (1970), Wood and Cahn (1977), and Iben and Truran (1978). Larger turnoff masses at a given age can arise from either a higher heavy-metal abundance or a reduced helium abundance $Y$. A difference in the mean $Y$ seems very unlikely as a causal factor because the galaxies would then have to have lower $Y$ than the globular clusters. An enhanced heavy-metal abundance in the galaxies appears insufficient to account for the required turnoff masses. The results of Ciardullo and Demarque (1977) show that even for 5 times solar metal abundance the red giant mass at 13 $\times 10^{9} \mathrm{yr}$ is only $1.02 M_{\odot}(Y=0.3)$. Thus, given the current state of knowledge of AGB evolution and mass-loss rates, the population of red, luminous stars which we suggest may be present in galaxies must originate from a parent population which is several billion years younger than the globular clusters.

How can one get a population of relatively young stars in an elliptical galaxy? One possibility is that this type of galaxy is younger in its entirety than previously believed. This hypothesis is probably not viable because of the difficulties which would then be encountered in reproducing various optical colors and line indices. Alternatively, star formation could have continued within a substantial fraction of the total volume of elliptical galaxies over a time interval $\sim 6$ $\times 10^{9} \mathrm{yr}$. Evidence that significant star formation has occurred over more than half of the lifetime of the dwarf elliptical M32 (at least in its nuclear region) has been presented by O'Connell (1980). Also, recall that age estimates for old disk groups stars such as used by Tinsley and Gunn in their models for early-type galaxies have typically been assigned ages of 5-6 $610^{9}$ years (e.g., Demarque and McClure 1977).

Finally, there remains the possibility that a tail of very metal-rich old stars (which are thus extremely red, but not extraordinarily luminous) could explain the color shifts seen between galaxies and globular clusters. This point will be discussed more quantitatively in a future publication.

To summarize: The differences in infrared colors and narrow-band indices between early-type galaxies and globular clusters could be explained by upper AGB stars contributing strongly to the near-infrared light. Higher masses seem to be required to produce stars more luminous than the first red-giant tip. If the Ciardullo-Demarque tracks are correct, and current wisdom on the question of mass loss in giant stars is at least approximately valid, then the galaxies could possess a significant fraction of stars with an age several billion years younger than that assigned to the galaxy as a whole.

\section{LUMINOSITIES OF GLOBULAR CLUSTERS}

An investigation of the luminosity distribution of globular clusters can yield information on the physical conditions that accompanied the formation of the stars in the clusters. The parameters whose values can be deduced are the mean mass of the globulars and the slope of the initial mass function as functions of metallicity or other variables. In our own Galaxy the known globulars are essentially a complete sample, so we can determine the mean luminosity as a function of metallicity. Values for the reddening and distance moduli are taken from Harris (1976), integrated $V$ photometry from Peterson and King (1975), and metallicities from Zinn (1980). We have derived the median $V$ values for bins in $[\mathrm{Fe} / \mathrm{H}]_{\text {Zinn }}$ and median $[\mathrm{Fe} / \mathrm{H}]_{\text {Zinn }}$ values for bins in $V$. Unfortunately, the typical bin contains only 16 globulars, and there is a large intrinsic dispersion in absolute magnitude. Nevertheless, the final result is that the median absolute visual magnitude shows no change with metallicity larger than $\pm 0.5 \mathrm{mag}$. A similar result is obtained for the median $\mathrm{K}$ magnitude as a function of metallicity. Any trend in absolute $\mathrm{K}$ magnitude is lost in the uncertainty in the median value.

An attempt to carry out such a procedure for M31 is fraught with difficulties as the sample of globulars observed is magnitude limited. We first consider just the sample reported here with infrared photometry, which is basically complete to $V=16$, and exclude those few globulars fainter than this limit.

The median $K$ magnitude increases slightly with increasing color, but the large uncertainty is due to the small number of clusters in each bin. Also, the result may not be physically significant because the lower bound is constant in $V$ rather than in $K$. An attempt to extend the sample by using the clusters in Vetesnik (1962) was inconclusive. To a first approximation, it appears that there is no gross trend (more than $\pm 0.7 \mathrm{mag}$ ) in either the median $V$ or $K$ magnitudes as function of color for the M31 globulars, in agreement with the results for our Galaxy. We note Harris and Racine (1979) have found that the luminosity function of the galactic and M31 globular systems are identical to within their uncertainties.

Given these results, can we set limits on the masses of globulars? Figure 8 shows the predicted integrated $K$ magnitudes for globular clusters with a total mass in stars of $10^{4} M_{\odot}$, and the values of metallicity and mass function used for the models of Appendix B. For dwarf-dominated models of a fixed total mass the dependence of $K$ integrated magnitude on metallicity is 


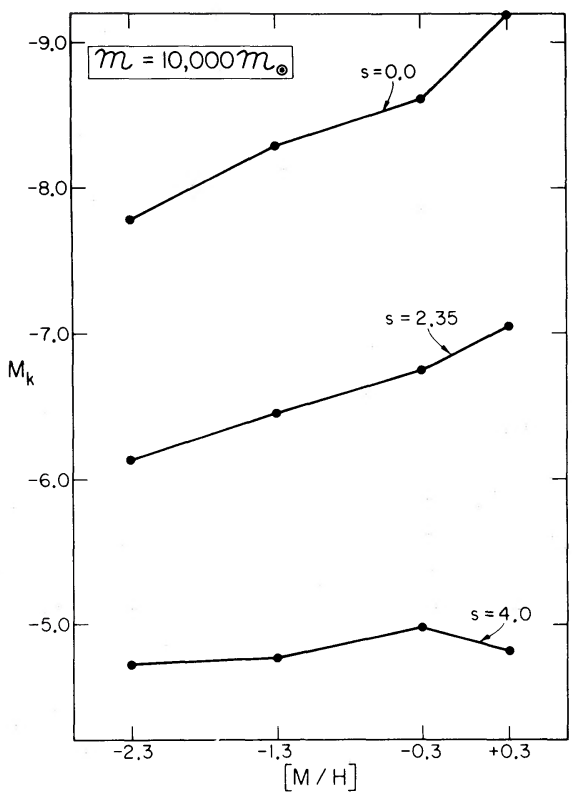

FIG. 8.-The integrated $\mathrm{K}$ magnitudes calculated from the models of Appendix B for globular clusters with constant total stellar mass of $10^{4} M_{\odot}$ are shown as functions of metallicity and initial mass function.

less than 0.4 mag over the total range in metallicity. For giant-dominated light and a constant total mass, $V$ becomes slightly fainter and $K$ somewhat brighter as the metallicity is increased (less than $1.0 \mathrm{mag}$ change from the most metal-poor model to a metal-rich model).

Changes in the slope of the initial mass function, $s$, have a much more profound effect on the luminosity than changes in the metallicity (see Fig. 8). It is intuitively clear that as $s$ is increased (i.e., more dwarfs relative to giants) for a fixed total mass, the total luminosity of the system must become fainter. This effect is large, even at small values of $s$, where, because the integrated light is always giant dominated, the colors are not affected. Changing $s$ from 0 to 2.35 decreases the integrated $V$ or $K$ magnitude by $2 \mathrm{mag}$; further increasing $s$ from 2.35 to 4.0 decreases the $V$ magnitude by approximately $2.5 \mathrm{mag}$. Thus from the observed constancy of the median $V$ and $K$ magnitudes, one may infer that a constant mean total mass and a constant mean $s$, independent of metallicity, are consistent with the data for the M31 and the galactic globulars. Also consistent with the data are a family of solutions, including $s$ increasing with metallicity from 0 to 2.35 and mean mass increasing by a factor of 2 as metallicity increases, or $s$ decreasing with metallicity from 2.35 to 0 and a mean mass decreasing by a factor of 10. Thus the correlation between $s$ and metallicity for globulars suggested by Da Costa (1977), if true, requires a strong correlation between mean mass and metallicity.

Metal-enhanced star formation as described by Talbot and Arnett (1973) is also qualitatively consistent with the data in that stars form more efficiently in more metal-rich systems, which for a fixed initial mass may thus have a larger total mass of stars and hence be better able to retain gas polluted by the first generation of supernovae, becoming even more metal rich. In addition, the Jeans mass is lower, so more dwarfs may be formed. After the leftover gas is lost, the mean total stellar mass and $s$ would both increase with metallicity, which is in qualitative agreement with the deduced constant median integrated $V$ and $K$ magnitudes of globulars.

Consideration of the galactic globular cluster system as a whole suggests that the case of constant mean mass and constant $s$ for the clusters irrespective of metallicity is close to the true case. The observed standard deviation in $V$ magnitude for the total sample of galactic globulars is $1.2 \mathrm{mag}$ (Alcaino 1979; Harris and Racine 1979). This can arise as previously discussed from variations in $s$ or from variations in the total mass of the clusters. Ignoring the latter, we find the maximum standard deviation in $s$ is approximately 1.0; while ignoring the former, we find the maximum standard deviation in total mass is a factor of 3 . Since any smaller variation in the total mass of galactic globulars seems physically unreasonable, and since any dispersion in $s$ will, as noted above, cause a large dispersion in integrated magnitude, there does not seem to be room for large variations in $s$.

\section{SUMMARY}

Infrared observations of 40 globular clusters in M31 are presented. The individual cluster reddenings, most of which are due only to foreground material in our Galaxy, have been taken from Searle (1980). The main results of this study are as follows.

1. The $(V-K)_{0}$ values for the M31 clusters are well correlated with other broad-band colors, such as $(U-V)_{0}$, and also with Searle's (1980) metallicity parameters $Q_{K}$ and $q$. The scatter in the $(V-K)_{0}$, $Q_{K}$ relationship is consistent with that expected from observational uncertainty alone.

2. The broad-band optical and infrared colors and $\mathrm{CO}$ and $\mathrm{H}_{2} \mathrm{O}$ indices of the M31 clusters are indistinguishable from those of galactic globulars.

3. Comparison of the M31 cluster data with similar data for early-type galaxies shows that galaxies extend to redder colors than the most metal-rich globulars. Also, early-type galaxies have, on average, redder $(V$ $-K)_{0}$ colors at a given $(U-V)_{0}$ and stronger $\mathrm{CO}$ and $\mathrm{H}_{2} \mathrm{O}$ indices at a given $(V-K)_{0}$. New data for a faint dwarf galaxy extend the region over which these differences are manifest.

4. New models are presented for the integrated light of old stellar systems. These are analogous to the models of ACMM but incorporate recent changes to the temperature and metallicity scales of globular cluster giants. Models with an initial mass function slope $\lesssim$ the Salpeter value agree quite well with the colors of the globular clusters, but diverge from the colors of early-type galaxies. 
5. We propose that the observed photometric differences between galaxies and globulars and between the galaxies and the models arise at least in part from the presence in the galaxies of a population of red, luminous giants and asymptotic giant-branch stars whose main-sequence precursors had masses in the range 1-2 $M_{\odot}$. An immediately obvious implication of this is that significant star formation in early-type galaxies has occurred over perhaps half of their lifetime. The deduced mass of the AGB precursors is quite uncertain, though, as it depends on our poor understanding of $\mathrm{AGB}$ evolution itself and on uncertain estimates for mass loss from stars on the GB and AGB.

6. The luminosities of the M31 and galactic globulars are discussed within the framework of the new models. Although the mean luminosity is independent of metallicity, the average total mass and average initial mass function as functions of metallicity are coupled and cannot be derived separately.

J. A. F. is grateful to the Director of the Hale Observatories for Guest Investigator privileges without which this project could not have been carried out. We thank L. Searle for many fruitful conversations and his continuing interest in this program. M. Aaronson was kind enough to provide us with tabulations of his unpublished data. D. Rabin pointed out some of the numerical problems in the Yale tracks. We particularly appreciate the assistance of S. Beckwith in obtaining some of the observations discussed here. Several of our colleagues at CTIO and Hale have made useful comments on a draft of this paper. This work was supported in part by NSF grants AST 77-20516 and AST 76-22676 and NASA grant NGL 05-002-207.

\section{APPENDIX A}

\section{CO AND $\mathrm{H}_{2} \mathrm{O}$ MEASUREMENTS OF THE VIRGO DWARF GALAXY}

It is of interest to obtain measurements of the narrow-band $\mathrm{CO}$ and $\mathrm{H}_{2} \mathrm{O}$ indices of the intrinsically faintest early-type galaxies for which we have broadband colors (Papers I and III). These objects, e.g., NGC 205, NGC 404, and the dwarf galaxy companions to the spiral galaxy M100, have $(U-V)_{0}$ and $(V$ $-K)_{0}$ colors in the range of those of intermediate metal-abundance globular clusters. The $\mathrm{CO}$ and $\mathrm{H}_{2} \mathrm{O}$ indices of these galaxies can give information about the latest type stars that these galaxies have managed to produce. The ultimate goal is to model the H-R diagram of these red stars, and to thus explain their origin in terms of metallicity and/or age. Proper interpretation of this kind of datum cannot as yet be given, but we should be able to show whether or not a faint blue galaxy contains any very red stars, such as must exist in the more luminous systems.

$\mathrm{M} 100 \mathrm{CH} 1$ = NGC 4328 (CH 1 refers to galaxy No. 1 on the finding chart of Sandage 1972) is one of the faint dwarf galaxy companions of the giant spiral M100 $=$ NGC 4321. Its broad-band $U-B$ and $B-V$ colors were given by Sandage (1972), and the broad- band infrared data were presented in Paper III. The advantage of measuring this galaxy over NGC 205, for example, is that we can sample a much greater fraction of the total light and are not therefore as subject to confusion by radial gradients in color or band strength.

The observations were carried out on the $5 \mathrm{~m}$ Hale telescope and the $2.5 \mathrm{~m}$ du Pont telescope, using observational techniques that are described fully in Papers I, II, and III. The results are given in Table 3. The observations were made on several nights, and the individual measurements, each of the same integration time, were averaged with equal weight. The distribution of these individual measurements about the mean was not quite Gaussian but was wider, flatter, and slightly skewed. Nevertheless, the individual statistical errors are nearly consistent with the dispersion of the measurements; the true dispersion is about 1.5 times as wide as expected. During each set of measurements of M100 CH 1 the nucleus of either NGC 4472 or NGC 4486 was measured as a local standard. In all cases these latter data were consistent with values

TABLE 3

$\mathrm{CO}$ AND $\mathrm{H}_{2} \mathrm{O}$ DATA FOR M100 $\mathrm{CH} 1$

\begin{tabular}{clcccrr}
\hline \hline Index & Telescope & $\begin{array}{c}\mathrm{Ap}^{\mathrm{a}} \\
(\operatorname{arcsec})\end{array}$ & $N^{\mathrm{b}}$ & Raw & Corrected $^{\mathrm{c}}$ & $\sigma_{m}$ \\
\hline $\mathrm{CO} \ldots \ldots \ldots \ldots \ldots \ldots \ldots \ldots$ & Hale & 15 & 14 & 0.105 & 0.13 & 0.02 \\
$\mathrm{H}_{2} \mathrm{O} \ldots \ldots \ldots \ldots \ldots \ldots$ & du Pont & 15 & 26 & 0.095 & 0.10 & 0.02 \\
\hline
\end{tabular}

a Aperture diameter.

${ }^{\mathrm{b}} N=$ number of individual color measurements. Average $\sigma_{m}$ per measurement $=0.05$ for $\mathrm{CO}$ and 0.09 for $\mathrm{H}_{2} \mathrm{O}$.

${ }^{\mathrm{c}}$ Corrected for redshift. Assume the velocity of M100 and the correction for the $\mathrm{CO}$ index from Paper I. There is no redshift correction for $\mathrm{H}_{2} \mathrm{O}$. 
published in Papers I and II, and the data in Table 3 are on the same photometric system as discussed in those papers.

Table 3 also contains the redshift-corrected indices which have been plotted in Figures 3 and 4 of this paper. As can be seen from these figures, both the $\mathrm{H}_{2} \mathrm{O}$ and $\mathrm{CO}$ indices of this dwarf galaxy are stronger than those of globular clusters at the same $U-V$ or $V-K$. We conclude that the faintest measurable dwarf galaxies contain a population of red stars which have a strong $2.3 \mu \mathrm{m} \mathrm{CO}$ band and which dominate the infrared light near $2 \mu \mathrm{m}$.

\section{APPENDIX B}

\section{MODIFICATIONS TO THE MODELS OF INTEGRATED COLORS FOR GLOBULAR CLUSTERS}

Aaronson et al. (1978, hereafter ACMM) presented a set of theoretical models for integrated colors of globular clusters. These models were based on the Ciardullo and Demarque (1977) isochrones with a single burst of star formation 13 billion years ago and a helium content by mass of $30 \%$. Models for four metallicities were calculated. The parameters of these models were calibrated using data then available for stars in globular clusters and in the field as described in ACMM.

Two recent additions to the fundamental data for late-type field and globular-cluster stars have required us to make modifications to the original models. The lunar interferometric studies of Ridgway et al. (1980) have established that the spectral-type (or, equivalently, $V-K$ color)-effective temperature calibration of Johnson (1966b) is up to $400 \mathrm{~K}$ too cool for giants cooler than $4000 \mathrm{~K}$. The ACMM models used the model atmospheres of Cohen, Frogel, and Persson (1978) for $T_{\text {eff }}$ between 4000 and $5000 \mathrm{~K}$, and used a slightly modified Johnson (1966b) scale cooler than that. Thus the model giant branches are too blue for a given $T_{\text {eff }}$ at very cool temperatures. This problem affects only the metal-rich models where there are such cool giants.

The second problem is the revised scale for cluster metallicities mentioned in $\S$ III $b$. In the ACMM models, the Ciardullo and Demarque isochrones for a metallicity of half-solar were slid along the $T_{e}$ axis and forced to fit the 47 Tuc giant and subgiant observations to remove problems in the isochrones arising from uncertainty in the convective mixing length. This temperature shift, $\Delta T$, was assumed to be a constant by ACMM because the M92 observations of CFP were fairly well produced with the $[\mathrm{M} / \mathrm{H}]=-2.3$ isochrone and the same shift as applied to 47 Tuc. It now appears that a more metal-poor isochrone should be fitted to the 47 Tuc observations. Because of the good fit to the M92 data, changing $\Delta T$ for 47 Tuc implies that the same $\Delta T$ cannot be used for all metallicities. Thus we use the original ACMM $\Delta T$ for $[\mathrm{M} / \mathrm{H}]=-2.3$ (based on the reasonable fit to $\mathrm{M} 92$ ), and derive a new $\Delta T$ for $[\mathrm{M} / \mathrm{H}]=-1.3$ from the M71 and 47 Tuc observations (Frogel, Persson, and Cohen 1979, 1980), and for $[\mathrm{M} / \mathrm{H}]=-0.3$ from M67 observations (CFP; Frogel, Persson, and Cohen 1980). This latter value of $\Delta T$ is also used for the isochrone with twice solar metallicity.
In addition, a numerical error has been discovered in the Ciardullo and Demarque (1977) bolometric luminosity functions. The most luminous magnitude interval in nearly all cases where a giant branch is included contains too many stars, often 10 times as many as in the previous (same sized) magnitude interval. The source of this problem is unclear, but could be numerical roundoff of the very slowly changing (as a fraction of $M_{\text {bol }}$ ) mass of the giants near the tip for a fixed age. The treatment adopted here has been to throw out the stars in the discordant box, and replace them with the same number of stars as in the next lower luminosity interval. The effect of this "last box problem" on indices shortward of $1 \mu \mathrm{m}$ is small, but the effect on $V-K$ is not negligible, as the superfluous giants are very red and very bright.

We have recalculated the ACMM models with these three changes; namely, the color-effective temperature relationship for $\mathbf{M}$ giants has been altered, the metallicity of 47 Tuc has been lowered, and the "last box problem" has been circumvented. Neither of the changes in the fundamental data affects the most metal-poor case, but the "last box problem" does make the new models have slightly bluer $V-K$ colors than the ACMM models. The new model results are given in Table 4. The horizontal branches (abbreviated HB) are those described by ACMM, but the ratio of $\mathrm{HB}$ stars to red giants, $n_{\mathrm{HB}} / n_{\mathrm{RG}}$, has been increased for the $[\mathrm{M} / \mathrm{H}]=-1.3$ model following the example of 47 Tuc (Lee 1977). Because the $U$ flux is predominantly from the turnoff and horizontal branch stars, it is independent of the temperatures adopted for the $\mathbf{M}$ giants. However, the change in metallicity scale affects the ultraviolet excess calibration used for the turnoff and main-sequence stars in a manner that is difficult to evaluate quantitatively. This change makes the $U-V$ colors redder. Note that because the metal-rich giant branches are significantly redder than those of ACMM, the importance of correctly modeling the $\mathrm{HB}$ (and the $U V$ excess for stars in the turnoff region) in order to predict the $U-V$ color is increased. Although the HB of the half-solar model does approximately reproduce that of $\mathrm{M} 67$, there is no empirical evidence in the twice solar case for positioning the $\mathrm{HB}$; uncertainties in the ratio of $\mathrm{HB}$ to red giant stars are just as serious. A further problem arises because M67 could be younger than globular clusters. Although the 
TABLE 4

Colors and Indices of the Population Models ${ }^{\text {a }}$

\begin{tabular}{|c|c|c|c|c|c|c|}
\hline $\begin{array}{c}{[\mathrm{M} / \mathrm{H}]} \\
\mathrm{HB} \\
n_{\mathrm{HB}} / n_{\mathrm{RG}}\end{array}$ & $s$ & $U-V$ & $V-K$ & $J-K$ & $\mathrm{CO}$ & $\mathrm{H}_{2} \mathrm{O}$ \\
\hline $\begin{array}{r}-2.3 \ldots \ldots \ldots \ldots \ldots \ldots \ldots \ldots \\
\quad \mathrm{BHB} \ldots \ldots \ldots \ldots \ldots \ldots \ldots \ldots \\
+1.0 \ldots \ldots \ldots \ldots \ldots \ldots \ldots\end{array}$ & $\begin{array}{l}0.0 \\
2.35 \\
4.04\end{array}$ & $\begin{array}{l}0.57 \\
0.58 \\
0.69\end{array}$ & $\begin{array}{l}1.77 \\
1.93 \\
2.69\end{array}$ & $\begin{array}{l}0.50 \\
0.55 \\
0.72\end{array}$ & $\begin{array}{l}+0.002 \\
-0.001 \\
-0.019\end{array}$ & $\begin{array}{l}0.035 \\
0.041 \\
0.063\end{array}$ \\
\hline 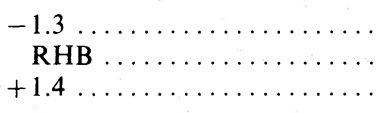 & $\begin{array}{l}0.0 \\
2.35 \\
4.0\end{array}$ & $\begin{array}{l}0.94 \\
0.91 \\
0.99\end{array}$ & $\begin{array}{l}2.34 \\
2.43 \\
3.14\end{array}$ & $\begin{array}{l}0.66 \\
0.67 \\
0.75\end{array}$ & $\begin{array}{l}+0.035 \\
+0.022 \\
-0.013\end{array}$ & $\begin{array}{l}0.043 \\
0.048 \\
0.078\end{array}$ \\
\hline $\begin{array}{r}-0.3 \ldots \ldots \ldots \ldots \ldots \ldots \ldots \ldots \\
\quad \operatorname{RHB} \ldots \ldots \ldots \ldots \ldots \ldots \ldots\end{array}$ & $\begin{array}{l}0.0 \\
2.35 \\
4.0\end{array}$ & $\begin{array}{l}1.39 \\
1.39 \\
1.57\end{array}$ & $\begin{array}{l}2.90 \\
2.94 \\
3.66\end{array}$ & $\begin{array}{l}0.81 \\
0.79 \\
0.82\end{array}$ & $\begin{array}{l}+0.125 \\
+0.092 \\
+0.012\end{array}$ & $\begin{array}{l}0.071 \\
0.079 \\
0.135\end{array}$ \\
\hline $\begin{array}{r}+0.3 \ldots \ldots \ldots \ldots \ldots \ldots \ldots \ldots \ldots \\
\quad \operatorname{RHB} \ldots \ldots \ldots \ldots \ldots \ldots \ldots \ldots \ldots\end{array}$ & $\begin{array}{l}0.0 \\
2.35 \\
4.0\end{array}$ & $\begin{array}{l}1.89 \\
1.83 \\
1.86\end{array}$ & $\begin{array}{l}3.52 \\
3.48 \\
4.03\end{array}$ & $\begin{array}{l}0.97 \\
0.93 \\
0.92\end{array}$ & $\begin{array}{l}+0.215 \\
+0.183 \\
+0.070\end{array}$ & $\begin{array}{l}0.157 \\
0.163 \\
0.251\end{array}$ \\
\hline
\end{tabular}

${ }^{a}$ This table is described in Appendix B and is a revision of Table 3 in ACMM. The slope of the initial mass function is $x=s-1$, where the Salpeter value is $s=2.35$. The $(J-K)$ colors are on the system of this paper and Paper I, which is not that used in ACMM.

effect of this on the giant branches can be evaluated, the movement of the $\mathrm{HB}$ with age is more uncertain. Because of these problems, it is best to regard the $U-V$ color as uncertain by \pm 0.10 mag for the metalrich cases; $V-K$ is uncertain by less than $\pm 0.10 \mathrm{mag}$ (due to the "last box problem" and the M67 age problem), and $J-K$ is unaffected.

The narrow-band indices have been calculated as in ACMM. A MMF value (see ACMM for definition) of 0.4 has been used for $\mathrm{H}_{2} \mathrm{O}$, which was the value used to construct the original models in ACMM. The predicted $\mathrm{H}_{2} \mathrm{O}$ index for the twice solar model has a considerable $( \pm 5 \%)$ uncertainty because of the uncertainty in the adopted MMF value.

The revised metallicities for 47 Tuc and M71, as well as the realization that mixing may have occurred in many stars near the red giant tip (e.g., Bell, Dickens, and Gustafsson 1979), suggest that the CO index treatment used in ACMM needs to be modified. This is because the calibration of the $\mathrm{CO}$ index as a function of metallicity was partially based on the observations of $\mathrm{CO}$ indices for globular cluster giants in M92 and M71 by CFP and Frogel, Persson, and Cohen (1979). A recalibration of the $\mathrm{CO}$ index, although needed, has not been attempted. The problem is such that the dependence of $\mathrm{CO}$ index on metallicity is now too strong in the models. One thus expects the predicted $\mathrm{CO}$ indices for models with metallicities much below solar to be too small compared to the observed values, as is indeed seen in Figure 3. The twice solar metallicity model will have a predicted $\mathrm{CO}$ slightly too large, but not by more than $2 \%$ based on tests made using the isochrones appropriate for a metallicity twice solar with the $\mathrm{CO}$ index assumed to behave as in the solar metallicity case as a function of $T_{\text {eff }}$. The most metalpoor model at $[\mathrm{M} / \mathrm{H}]=-2.3$ is also not significantly affected. (Its $\mathrm{CO}$ index should perhaps be $0.00-$ $0.02 \mathrm{mag}$ larger.) This is because the metallicity is so low that even though the strength of the $\mathrm{CO}$ absorption has been underestimated, $\mathrm{CO}$ will still be essentially absent in most of the stars, so that the CO index will in any case be that of a blackbody at the appropriate $T_{\text {eff }}$.

\section{REFERENCES}

Aaronson, M., Cohen, J. G., Mould, J., and Malkan, M. 1978, Ap. $J ., 233,823$ (ACMM).

Aaronson, M., Frogel, J. A., and Persson, S. E. 1978, Ap. J., 220, 442 (Paper II).

Alcaino, G. 1979, Vistas in Astronomy, 23, 1.

Bell, R. A., Dickens, R. J., and Gustafsson, B. 1979, Ap. J., 229, 604. Canterna, R., and Schommer, R. A. 1978, Ap. J. (Letters), 219, L119.

Ciardullo, R. B., and Demarque, P. 1977, Trans. Astr. Obs. Yale Univ., 35.

- 1978, in IAU Symposium No. 80, The H-R Diagram, ed. A. G. D. Philip and D. S. Hayes (Dordrecht: Reidel), p. 345.

Cohen, J. G., Frogel, J. A., and Persson, S. E. 1978, Ap. J., 222, 165 (CFP).

Cohen, J. G. 1979, IAU Symposium No. 85, Star Clusters (Dordrecht: Reidel), in press.
Cowley, A. P., Hartwick, F. D. A., and Sargent, W. L. W. 1978, Ap. J., $220,453$.

Da Costa, G. 1977, Ph.D. thesis, Australian National University.

Demarque, P., and McClure, R. D. 1977, in Evolution of Galaxies and Stellar Populations, ed. B. M. Tinsley and R. B. Larson (New Haven: Yale University Observatory), p. 99.

de Vaucouleurs, G., de Vaucouleurs, A., and Corwin, H. G., Jr. 1976, Second Reference Catalogue of Bright Galaxies (Austin: University of Texas Press).

Faber, S. M. 1973, Ap. J., 179, 731.

1977, in Evolution of Galaxies and Stellar Populations, ed. B. M. Tinsley and R. B. Larson (New Haven: Yale University Observatory), p. 157.

Freeman, K. C. 1977, in Evolution of Galaxies and Stellar Populations, ed. B. M. Tinsley and R. B. Larson (New Haven: Yale University Observatory), p. 133. 
Frogel, J. A., Persson, S. E., Aaronson, M., and Matthews, K. 1978, Ap. J., 220, 75 (Paper I)

Frogel, J. A., Persson, S. E., and Cohen, J. G. 1978, Proc. NATO Conf. on Globular Clusters, Cambridge, England.

- 1979, Ap. J., 227, 499. $1980, A p . J$. , to be submitted.

Gingold, R. A. 1974, Ap. J., 193, 177.

Hanes, D. A. 1977, M.N.R.A.S., 179, 331.

Harris, H. C., and Canterna, R. 1977, A.J., 82, 798.

Harris, W. E. 1974, Ph.D. thesis, University of Toronto. 1976, A.J., 81, 1095.

Harris, W. E., and Racine, R. 1979, Ann. Rev. Astr. Ap., 17, 246.

Iben, Jr., I., and Truran, J. W. 1978, Ap. J., 220, 980.

Johnson, H. L. 1966a, in Nebulae and Interstellar Matter, ed. B. M Middlehurst and L. H. Aller (Chicago: University of Chicago Press), p. 167.

1966b, Ann. Rev. Astr. Ap., 4, 193.

Larson, R. B., and Tinsley, B. M. 1978, Ap. J., 219, 46.

Lee, S. W. 1977, Astr. Ap. Suppl., 27, 381.

McClure, R. D., and Racine, R. 1969, A.J., 74, 1000.

Mould, J. R., and Aaronson, M. 1979, Ap. J., 232, 421.

O'Connell, R. W. 1976, Ap. J., 206, 370 (OC). 1980, Ap. J., 236, 430.

Persson, S. E., Frogel, J. A., and Aaronson, M. 1979, Ap. J. Suppl., 39, 61 (Paper III).

Persson, S. E., Frogel, J. A., Cohen, J. G., Aaronson, M., and Matthews, K. 1980a, Ap. J., 235, 452. 1980b, Ap. J., to be submitted.
Peterson, C. J., and King, I. R. 1975, A.J., 80, 427.

Pilachowski, C. A., Canterna, R., and Wallerstein, G. 1980, Ap. J. (Letters), 235, L21.

Ridgway, S. T., Joyce, R. R., White, N. M., and Wing, R. F. 1980, Ap. J., 235, 126.

Rose, W. K., and Smith, R. L. 1970, Ap. J., 159, 903.

Sandage, A. 1972, Ap. J., 176, 21.

Sargent, W. L. W., Kowal, C. T., Hartwick, F. D. A., and van den Bergh, S. 1977, A.J., 82, 947.

Searle, L. 1978, talk presented at NATO Conference on Globular Clusters, Cambridge, England. . 1980, Ap. J., to be submitted.

Searle, L., and Zinn, R. 1978, Ap. J., 225, 357.

Spinrad, H., and Schweizer, F. 1972, Ap. J., 171, 403.

Strom, S. E., Strom, K. M., Goad, J. W., Vrba, F. J., and Rice, W. 1976, Ap. J., 204, 864.

Struck-Marcell, C., and Tinsley, B. M. 1978, Ap. J., 221, 562.

Talbot, R. J., Jr., and Arnett, W. D. 1973, Ap. J., 186, 69.

Tinsley, B. M. 1978, Ap. J., 222, 14.

Tinsley, B. M., and Gunn, J. E. 1976a, Ap. J., 203, 52 (TG). 1976b, Ap. J., 206, 525.

Tsuji, T. 1978, Astr. and Ap., 62, 29.

van den Bergh, S. 1967, A.J., 72, 70. . 1969, Ap. J. Suppl., 19, 145.

Vetesnik, M. 1962, Bull. Astr. Inst. Czechoslovakia, 13, 180

Wood, P. R., and Cahn, J. H. 1977, Ap. J., 211, 499.

Zinn, R. 1980, Ap. J. Suppl., 42, 19.

Judith G. Cohen: Astronomy Department, 105-24, California Institute of Technology, Pasadena, CA 91125

JAY A. Frogel: Cerro Tololo Inter-American Observatory, Casilla 63-D, La Serena, Chile

S. E. Persson : Mount Wilson and Las Campanas Observatories, 813 Santa Barbara Street, Pasadena, CA 91101 